\title{
A Cavity-Backed Rectangular Aperture Antenna With Application to a Tilted Fan Beam Array Antenna
}

\author{
Hisamatsu Nakano, Fellow, IEEE, Masatoshi Iwatsuki, Masanori Sakurai, and Junji Yamauchi, Member, IEEE
}

\begin{abstract}
A rectangular aperture of $A_{x} \times A_{y}$, cut in the top conducting plate of a triplate transmission line and backed by a cavity, radiates a tilted beam off the direction normal to the aperture. The mechanism of the radiation is explained using the Poynting vector distribution above the aperture and the phase distribution of the electric field over the aperture. The tilt angle is calculated as a function of side length $A_{x}$ for a representative value of $A_{y}=18 \mathrm{~mm}=0.747 \lambda_{12.45}$, where $\lambda_{12.45}$ is the wavelength at a test frequency of $12.45 \mathrm{GHz}$. A tilted beam of approximately $27^{\circ}$ is realized at $A_{x} / A_{y}=8 / 9$ with a gain of approximately $8 \mathrm{~dB}$. Using this value of $A_{x} / A_{y}$, an array antenna composed of rectangular cavity-backed aperture elements is investigated. The array forms a tilted fan beam without phase shifters. The frequency responses of the gain and input impedance are discussed.
\end{abstract}

Index Terms-Aperture antenna, finite-difference time-domain (FDTD) method analysis, tilted beam formation, triplate transmission line antenna.

\section{INTRODUCTION}

W HEN a square aperture is cut in the top conducting plate of a triplate transmission line (TTL) and fed by a probe, this structure acts as a radiation element [1], [2]. This radiation element is referred to as a square aperture antenna. The probe exciting the square aperture is the extension of a feed line located between the top and bottom plates of the TTL. An advantage of the square aperture antenna is that the feed line, by virtue of the closed structure of the TTL, does not affect the radiation pattern.

The square aperture in [2], whose side length is approximately one-half wavelength, radiates an axial beam, i.e., the maximum radiation is in the direction normal to the aperture plane. For mobile communications, a tilted beam is often required in addition to an axial beam. Recent investigation shows that the beam radiated from the square aperture antenna can be tilted from the direction normal to the aperture plane by increasing the size of the aperture [3]. However, an increase in aperture size is undesirable when constructing an array antenna composed of square aperture elements, because the distance between the array elements must also be increased, which gives rise to undesired grating lobes.

This paper presents a rectangular aperture antenna which overcomes the aforementioned grating lobe problem, where the rectangular aperture of short side length $A_{x}$ and long side length $A_{y}$ is backed by a cavity [4]. The cavity is used to improve the

Manuscript received August 14, 2001; revised January 5, 2002.

The authors are with the College of Engineering, Hosei University, Tokyo, 184-8584 Japan (e-mail: nakano@k.hosei.ac.jp).

Digital Object Identifier 10.1109/TAP.2003.811085 radiation efficiency, while still obtaining a tilted beam. Note that the rectangular aperture in this paper is designed to be a linear array antenna element (arrayed in the x-direction), and, therefore, the long side length $A_{y}$ is allowed to have a relatively large value $\left(A_{y}\right.$ is chosen to be approximately 0.75 wavelength, as seen later).

Analysis of the rectangular aperture antenna is performed using the finite-difference time-domain (FDTD) method [5]-[7]. Based on the fact that the antenna structure is symmetrical with respect to one plane ( $\mathrm{y}-\mathrm{z}$ plane), the computation space for the FDTD method is reduced to one-half of the full computation space. The smaller computation space reduces the computation time for the FDTD method.

First, the analysis results at a test frequency of $12.45 \mathrm{GHz}$, including the input impedance, gain, and beam direction as a function of aperture aspect ratio $A_{x} / A_{y}$, are summarized (Note: an aperture antenna with a fixed aspect ratio of $A_{x} / A_{y}=1$ (ranging from $A_{x}=0.25$ wavelengths to 0.75 wavelengths) is discussed in [3]). The mechanism for tilted beam formation is explained using the electric field distribution over the rectangular aperture. Frequency responses of the radiation characteristics are also summarized and discussed.

Second, the abovementioned results are used to construct an array antenna, where $N$ rectangular aperture elements are linearly arrayed to form a tilted fan beam without phase shifters. A representative tilted fan beam at a test frequency of $12.45 \mathrm{GHz}$ is presented. In addition, the frequency responses of the gain and input impedance for the $\mathrm{N}$-rectangular aperture array antenna are revealed over a frequency range of 10.65 to $14.25 \mathrm{GHz}$.

Throughout this paper, some experimental results are presented to confirm the theoretical results.

\section{CONFIGURATION}

Fig. 1 shows the configuration and coordinate system of a cavity-backed rectangular aperture antenna constructed using a TTL. The TTL has identical apertures of area of $A_{x} \times A_{y}$ in the top and bottom conducting plates. These top and bottom apertures are located symmetrically with respect to a plane parallel to the $\mathrm{x}-\mathrm{y}$ plane at $z=-B$. The bottom aperture is used to construct a rectangular cavity of depth $D_{\text {cav }}$. The space inside the cavity is filled with air.

The inner strip conductor of the TTL at $z=-B$ is embedded in a dielectric material of relative permittivity $\varepsilon_{r}$ and forms a feed line. A strip line probe of length $L_{\text {pro }}$ and width $W_{\text {pro, }}$, which is an extension of the feed line, excites the top aperture. For convenience, the plane at $z=-B$, parallel to the $\mathrm{x}-\mathrm{y}$ plane, is called the feed line plane throughout this paper. 


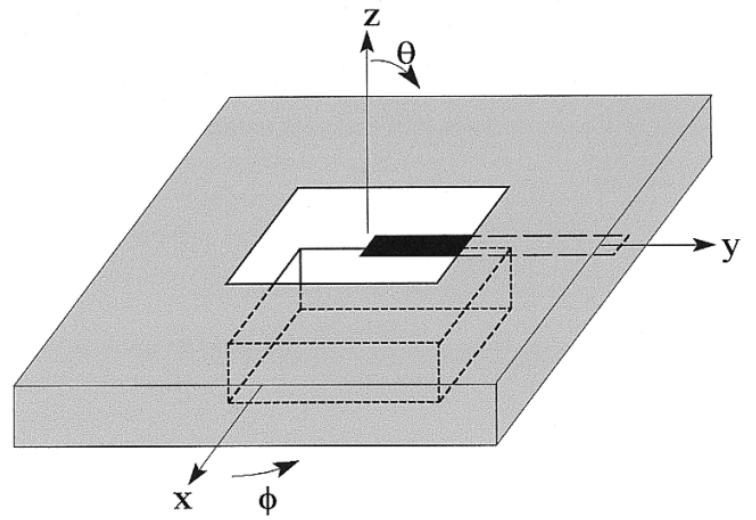

(a)

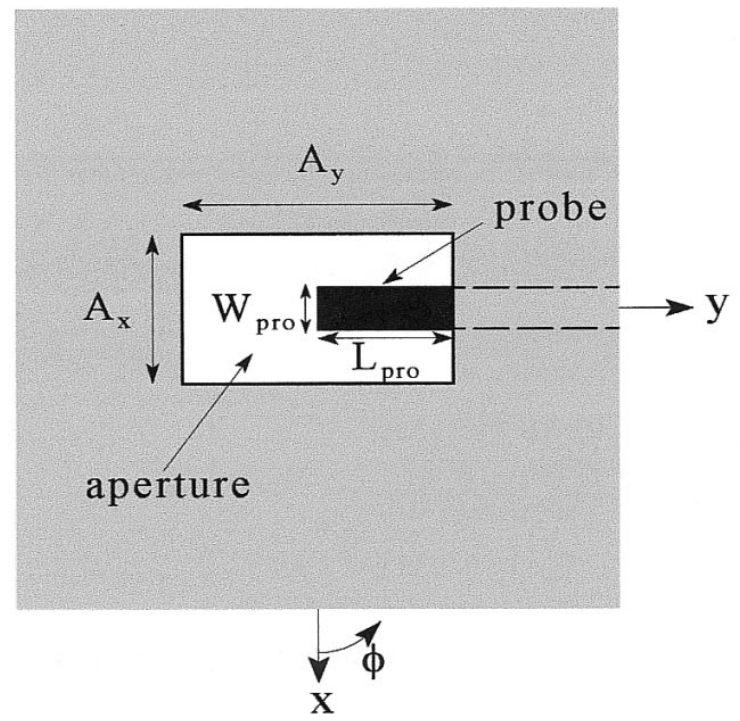

(b)

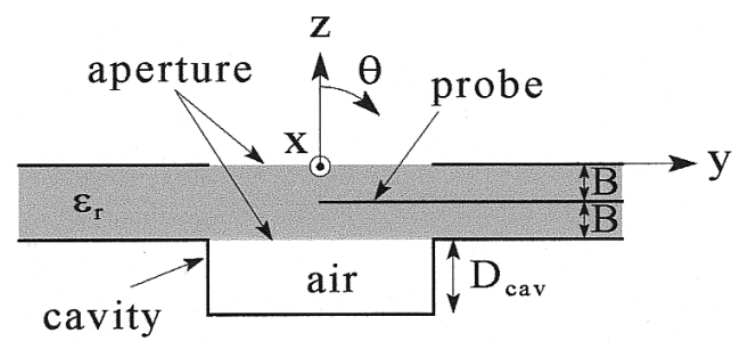

(c)

Fig. 1. Configuration of a rectangular aperture antenna backed by a cavity. (a) Perspective view. (b) Top view. (c) Cross-sectional view.

A frequency of $f_{0}=12.45 \mathrm{GHz}$ is chosen as a test (design) frequency. The following parameters are arbitrarily chosen and fixed: $A_{y}=18 \mathrm{~mm}=0.747 \lambda_{12.45}, B=2 \mathrm{~mm}=0.083 \lambda_{12.45}$, $\varepsilon_{r}=1.2, L_{\text {pro }}=A_{y} / 2=9 \mathrm{~mm}=0.373 \lambda_{12.45}, W_{\text {pro }}=$ $2 \mathrm{~mm}=0.083 \lambda_{12.45}$, and $D_{\text {cav }}=6 \mathrm{~mm}=0.25 \lambda_{12.45}$, where $\lambda_{12.45}(=24.1 \mathrm{~mm})$ is the free-space wavelength at the test frequency $f_{0}=12.45 \mathrm{GHz}$.

To simplify the following discussions, only the x-side length of the aperture $A_{x}$ (or an aspect ratio of $\left.A_{x} / A_{y}=A_{x} / 0.747 \lambda_{12.45}\right)$ is changed subject to the objectives of the analysis.

\section{ANTENNA CHARACTERISTICS}

The following assumptions are used: 1) the TTL is of infinite extent, i.e., the top and bottom plates are of infinite extent; 2 ) the conductor and dielectric materials for the TTL are perfectly conducting and lossless, respectively; and 3) the top, bottom, and inner plates of the TTL are infinitesimally thin. Based on these assumptions, the FDTD method [5], [6] with rectangular cells (Yee mesh) is used for the analysis of the aperture antenna. Yee's algorithm is programmed with Liao's second order absorbing condition [7].

The antenna is symmetrical with respect to the $y-z$ plane, as shown in Fig. 1. Therefore, the full analysis space $N_{x} \Delta x \times$ $N_{y} \Delta y \times N_{z} \Delta z$ can be reduced to $\left(N_{x} / 2\right) \Delta x \times N_{y} \Delta y \times N_{z} \Delta z$, where $N_{x}, N_{y}$, and $N_{z}$ are integers for specifying the full analysis space, and $\Delta x, \Delta y$, and $\Delta z$ are, respectively, Yee mesh lengths in the $\mathrm{x}, \mathrm{y}$, and $\mathrm{z}$ directions.

First, the antenna characteristics at a test frequency of $12.45 \mathrm{GHz}$ are presented in Figs. 2-5. Fig. 2 visualizes the power flow by using Poynting vectors, where the $\mathrm{x}$-side length of the aperture is chosen to be $A_{x}=16 \mathrm{~mm}=0.664 \lambda_{12.45}$, which leads to an aspect ratio of $A_{x} / A_{y}=8 / 9=0.89$. Fig. 2(a) shows the Poynting vectors in a plane parallel to the $\mathrm{x}-\mathrm{y}$ plane at $z=-B+(\Delta z / 2)=-2 \mathrm{~mm}+(0.5 \mathrm{~mm} / 2)=-0.0726 \lambda_{12.45}$, which is very close to the feed line plane located at $z=-B$. The Poynting vectors are distributed symmetrically with respect to the y axis due to the structural symmetry, and hence the radiation pattern in the $\mathrm{x}-\mathrm{z}$ plane $\left(\phi=0^{\circ}\right.$ plane) is symmetrical with respect to the $\mathrm{z}$ axis, as shown in Fig. 3(a).

Fig. 2(b) shows the Poynting vectors in the $y-z$ plane. The Poynting vectors above the aperture are not symmetrical with respect to the $\mathrm{z}$ axis. This means that the radiation beam is tilted from the $\mathrm{z}$ axis. The radiation pattern, Fig. 3(b), clearly shows this fact. The experimental radiation patterns are also presented in Fig. 3, showing good agreement with the theoretical results except for angles near $\theta=90^{\circ}$. This disagreement can be attributed to the finite size of the experimental antenna (note: to approximate the theoretical TTL structure of infinite extent, the dimensions of the top and bottom plates of the experimental antenna are chosen to be large with side lengths of $130 \mathrm{~mm}=5.39 \lambda_{12.45}$ in the $\mathrm{x}$ - and y-directions, and absorbing material is attached to the inside of the vertical walls surrounding the TTL).

The tilted radiation pattern of Fig. 3 (b) is related to the phase of the electric field component $E_{y}$ over the aperture. As shown in Fig. 4, the phase of $E_{y}$ for $A_{x} / A_{y}=8 / 9$ is delayed in the negative y-region, compared with that in the positive y-region. Due to the phase delay, the maximum radiation from the aperture exists in the negative $y-z$ plane. That is, the beam is tilted, as shown in Fig. 3 (b).

The side length $A_{x}$ affects the phase distribution of $E_{y}$ over the aperture, and changes the tilt angle of the radiation beam from the $\mathrm{z}$ axis. The solid line (labeled "with a cavity") in Fig. 5 shows the tilt angle $\theta_{t}$ as a function of aspect ratio $A_{x} / A_{y}$. It should be noted that the radiation beam in the $y-z$ plane has a wide half-power beam width (HPBW), as shown in Fig. 3 (b), and hence, $\theta_{\mathbf{t}}$ is evaluated as the average of two angles that de- 


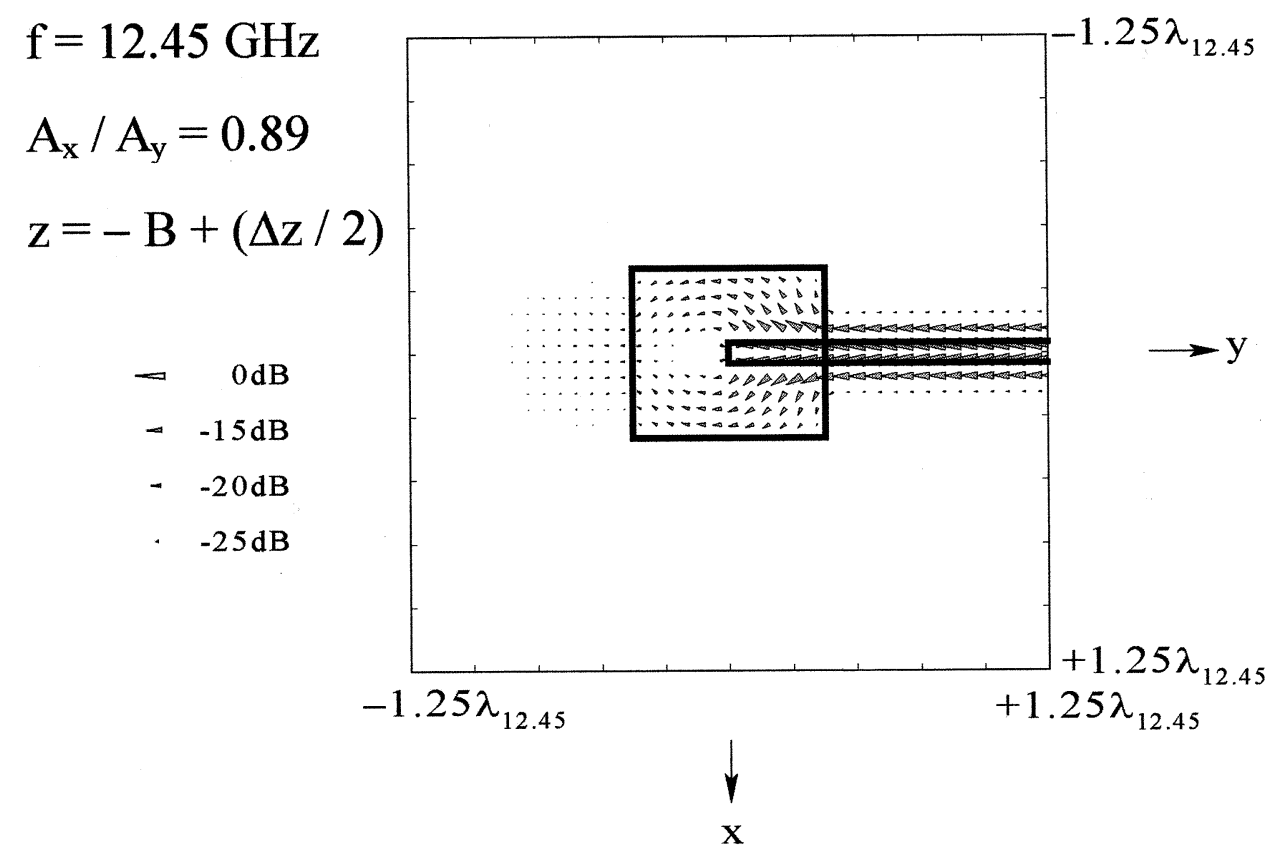

(a)

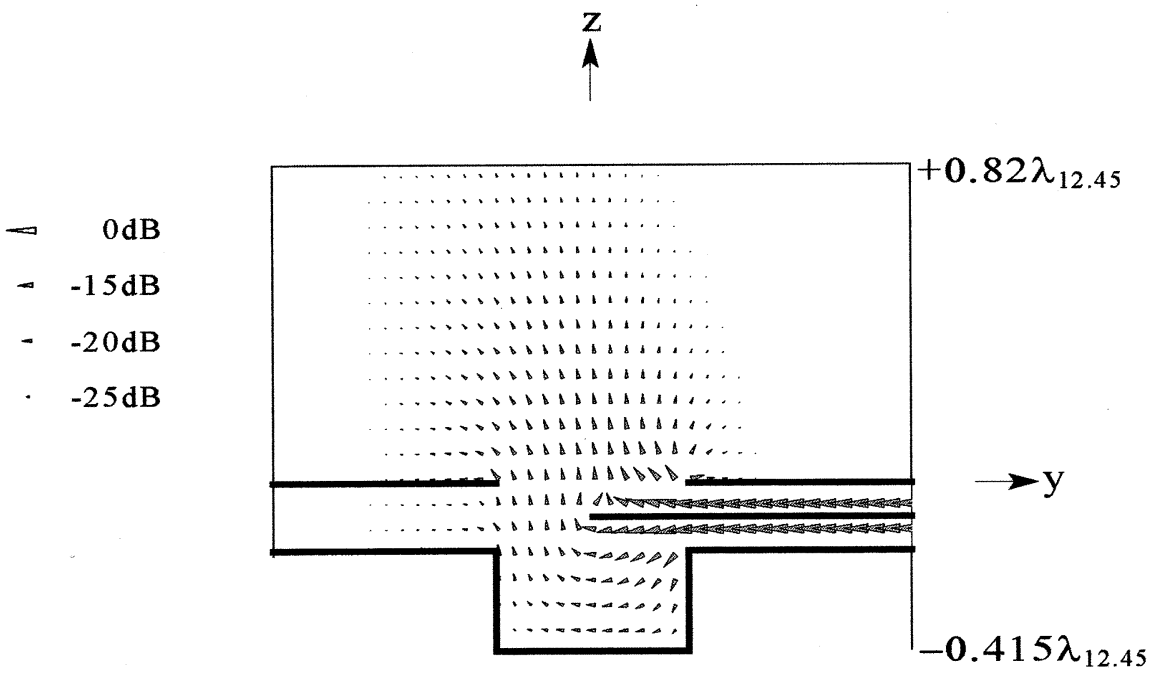

(b)

Fig. 2. Poynting vectors. (a) In a plane parallel to the $\mathrm{x}-\mathrm{y}$ plane at $z=-B+(\Delta z / 2)=-0.0726 \lambda_{12.45}$. (b) In the $\mathrm{y}-\mathrm{z}$ plane.

scribe the edges of the HPBW. It is found that the tilt angle $\theta_{t}$ increases as $A_{x} / A_{y}$ increases. A tilt angle of approximately $\theta_{t}=27^{\circ}$ is obtained at $A_{x} / A_{y}=0.89$ (=8/9 with $A_{x}=$ $\left.0.664 \lambda_{12.45}\right)$.

The input impedance (the impedance evaluated at the aperture edge $\left.\left(y=A_{y} / 2\right)\right), Z_{i n}=R_{i n}+j X_{i n}$, as a function of aspect ratio $A_{x} / A_{y}$ (=0.44 to 1.33$)$ is presented in [4]. Note that the variation in the resistance $R_{\text {in }}$ is relatively small in the vicinity of $A_{x} / A_{y}=0.89$. The gain in the beam direction $\left(\theta=\theta_{\mathrm{t}}\right.$ shown in Fig. $\left.5, \phi=270^{\circ}\right)$ and radiation efficiency as a function of aspect ratio $A_{x} / A_{y}$ are also shown in [4]. A gain of approximately $8 \mathrm{~dB}$ with a high radiation efficiency of approximately $\eta_{\mathrm{rad}}=87 \%$ is obtained at $A_{x} / A_{y}=0.89$. An interesting fact is that there is no significant decrease in the gain as $A_{x} / A_{y}$ increases (and correspondingly the tilt angle increases). This is due to fact that a gain increase resulting from the narrowed HPBW of the radiation pattern in the $\mathrm{x}-\mathrm{z}$ plane compensates for a gain decrease resulting from the widened HPBW of the tilted beam pattern in the $y-z$ plane.

For comparison, the tilt angle, gain in the beam direction, and radiation efficiency of a corresponding antenna without a cavity [a flat bottom structure (FBS) with $D_{\text {cav }}=0$ ] are also calculated in the same range from $A_{x} / A_{y}=0.44$ to 1.33 . As seen from the dashed line (labeled "without a cavity") in Fig. 5, the beam for the FBS is tilted in the positive $y-z$ plane. The gain in the beam direction for the FBS is approximately $6 \mathrm{~dB}$ at $A_{x} / A_{y}=0.89$ ( $2 \mathrm{~dB}$ lower than that of the cavity-backed structure), with a radiation efficiency of 66\% (21\% lower than that of the cavity-backed structure).

So far, the antenna characteristics at a test frequency of $12.45 \mathrm{GHz}$ have been summarized. Next, the frequency responses are revealed for a representative cavity-backed rectan- 


$$
\mathrm{f}=12.45 \mathrm{GHz}
$$

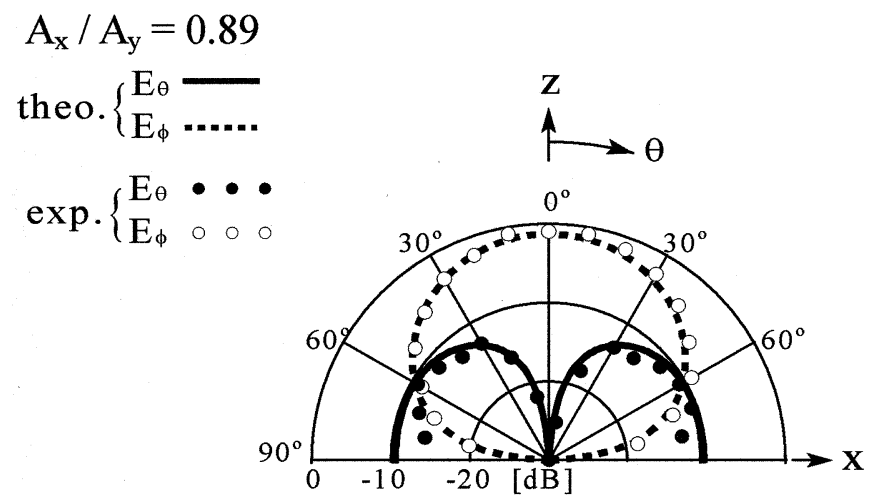

(a)

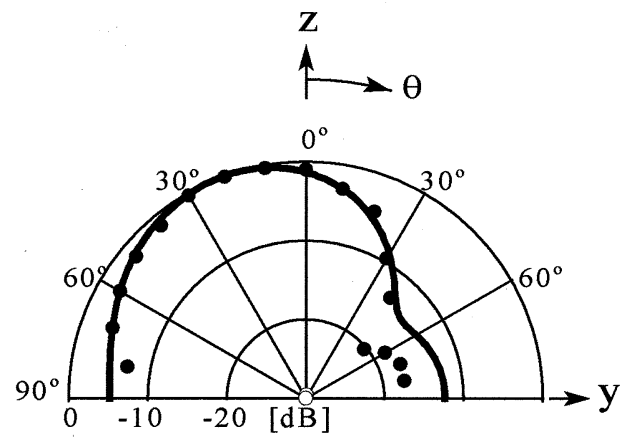

(b)

Fig. 3. Radiation patterns. (a) In the $x-z$ plane. (b) In the $y-z$ plane.

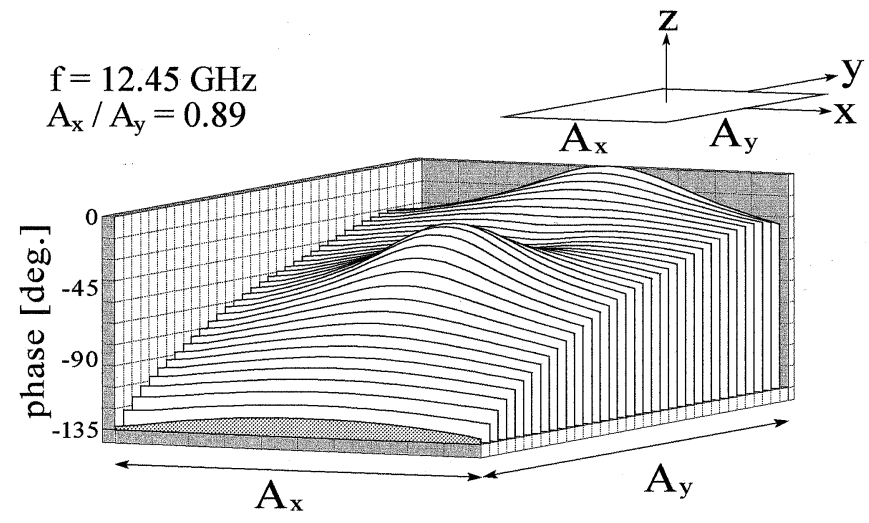

Fig. 4. Phase distribution of electric field $E_{y}$.

gular aperture antenna with an aspect ratio of $A_{x} / A_{y}=8 / 9=$ 0.89. Fig. 6 shows the frequency response of tilt angle $\theta_{t}$. As the frequency increases, the phase delay of $E_{y}$ in the negative $y$-region of the aperture increases, resulting in an increase in the tilt angle $\theta_{t}$. The tilt angle in this analysis range varies by $18^{\circ}$ (from $\theta_{t}=14^{\circ}$ at $10.65 \mathrm{GHz}$ to $32^{\circ}$ at $14.25 \mathrm{GHz}$ ). The theoretical results are confirmed by experimental results.

Figs. 7 and 8 show the input impedance $Z_{\text {in }}=R_{\text {in }}+j X_{\text {in }}$ and the gain (computed in the fixed direction $\left(\theta=27^{\circ}, \phi=\right.$ $\left.270^{\circ}\right)$ ) as a function of frequency, respectively. It is found that, as the frequency increases, $R_{\text {in }}$ decreases, while $X_{\text {in }}$ remains relatively constant. Note that the fixed direction $\left(\theta=27^{\circ}\right.$, $\phi=270^{\circ}$ ) is the beam direction at a frequency of $12.45 \mathrm{GHz}$. The gain variation is relatively small $( \pm 1 \mathrm{~dB}$ from $8 \mathrm{~dB})$. Also,

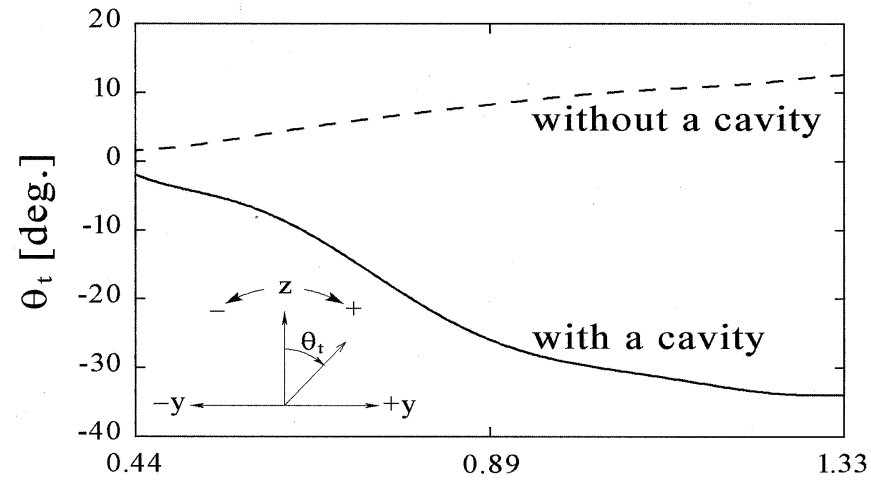

$\mathrm{A}_{\mathrm{x}} / \mathrm{A}_{\mathrm{y}}$

Fig. 5. Tilt angle $\theta_{t}$ as a function of $A_{x} / A_{y}$. The solid line shows $\theta_{t}$ for an aperture with a cavity and the dashed line shows $\theta_{t}$ for an aperture without a cavity.

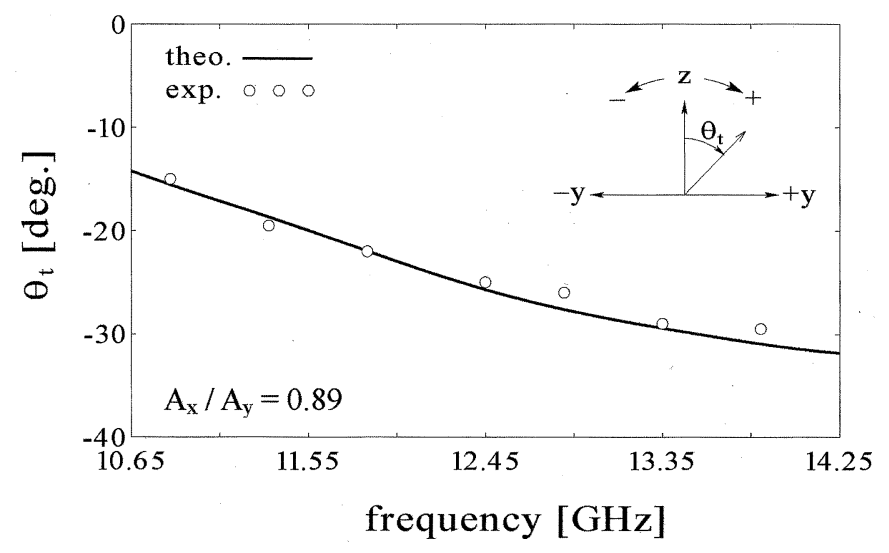

Fig. 6. Tilt angle $\theta_{t}$ as a function of frequency.

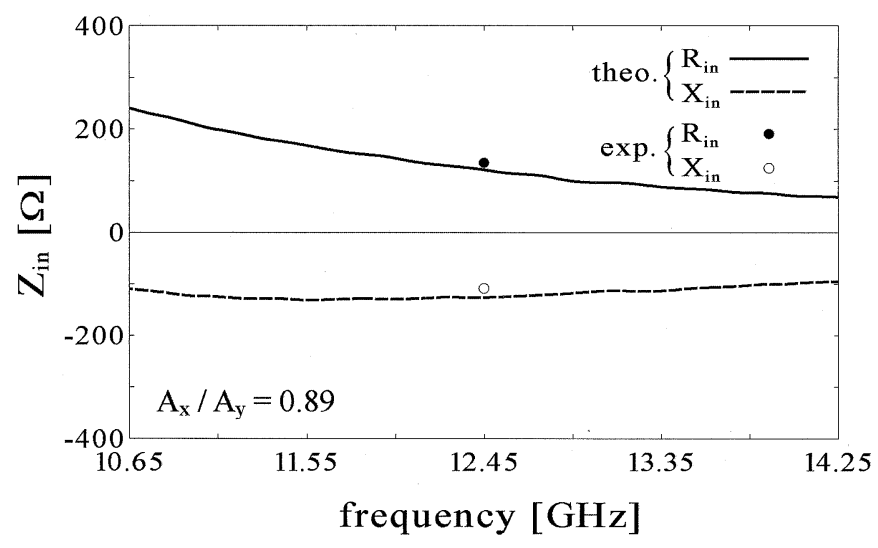

Fig. 7. Input impedance $Z_{\text {in }}=R_{\text {in }}+j X_{\text {in }}$ as a function of frequency.

note that the frequency response of the computed gain in the maximum beam direction $\left(\theta=\theta_{t}\right.$ in Fig. 6) is close to that in the fixed direction. The gain difference between these two cases is less than $0.5 \mathrm{~dB}$.

\section{ANTENNA ARRAYS FOR A FAN BEAM}

A wide beam tilted in the E plane ( $\mathrm{y}-\mathrm{z}$ plane) is obtained in Section III. In this section, an array antenna composed of $N$ rectangular aperture elements realizes a tilted fan beam without 


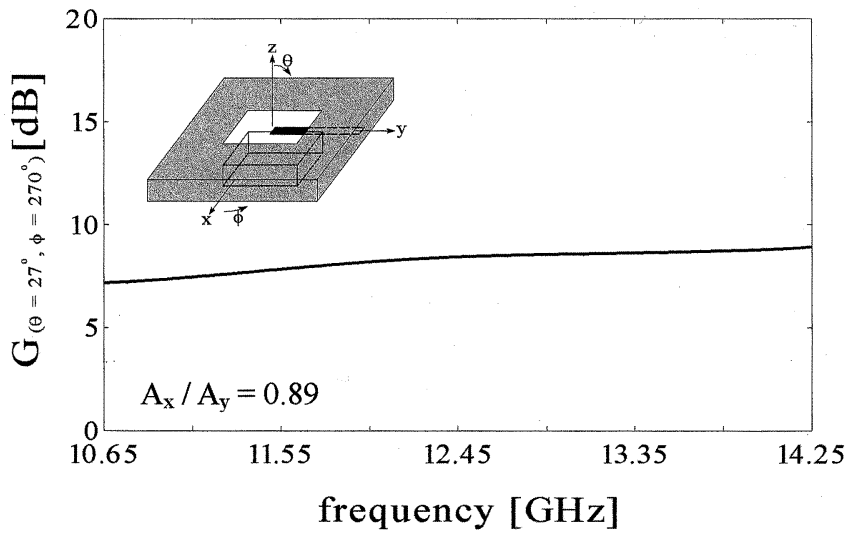

Fig. 8. Gain in the fixed direction $\left(\theta=27^{\circ}, \phi=270^{\circ}\right)$ as a function of frequency.

phase shifters: a sharpened radiation pattern in the $\mathrm{H}$ plane $(\mathrm{x}-\mathrm{Z}$ plane) and a tilted, wide radiation pattern in the $\mathrm{E}$ plane $(\mathrm{y}-\mathrm{z}$ plane). This tilted fan beam is useful for mobile-satellite communications in that the beam can avoid communication disruptions caused by movement (of automobile, ship, and so on) in the $y-z$ plane. Note that the fan beam is usually scanned mechanically in the $\mathrm{x}-\mathrm{y}$ plane.

Fig. 9 shows an array antenna composed of $N$ rectangular aperture elements located along the $\mathrm{x}$ axis, where $d_{x}$ denotes the array distance between the neighboring aperture elements (or the neighboring probes (from center to center)). Throughout this section, an aperture antenna with an aspect ratio of $A_{x} / A_{y}=$ 0.89 is chosen as an array element, with an array distance of $d_{x}=0.79 \lambda_{12.45}$. Other configuration parameters are as defined in Section II. Note that the chosen array element has a tilted beam of $\theta_{t}=27^{\circ}$ and a gain of approximately $8 \mathrm{~dB}$, as revealed in Section III.

As the number of array elements $N$ increases, the radiation pattern in the $\mathrm{x}-\mathrm{z}$ plane becomes narrower due to array effects, while the tilted, wide radiation pattern in the $\mathrm{y}-\mathrm{z}$ plane remains unchanged. Thus, the array forms a tilted fan beam with an increased gain. Fig. 10 shows representative radiation patterns for $N=4$.

Fig. 11 shows the gains for $N=2,4$, and 6 as a function of frequency. These gains are evaluated in the fixed direction $\left(\theta=27^{\circ}, \phi=270^{\circ}\right)$. Note that each gain is affected by three factors: the mutual coupling among the array elements, the loss due to generation of parallel-plate mode power, which propagates between the top and bottom plates of the TTL [2], [11], and the loss due to the dielectric and conducting materials used for the TTL [10]. The second and third factors obviously decrease the gain. The theoretical gain in this paper includes the effects of the mutual coupling and the loss due to parallel-plate mode power under the condition that the dielectric and conducting materials are lossless. In the experiment, a foam material, which has a low permittivity $\left(\varepsilon_{r}=1.2\right)$ with an extremely small loss tangent (on the order of $\tan \delta=0.001$ ) is used to approximate the assumption of the lossless dielectric, by which the feed lines of copper (the length from the generator to the edge of the aperture is not long (approximately $85 \mathrm{~mm}=3.53 \lambda_{12.45}$ )) are supported.

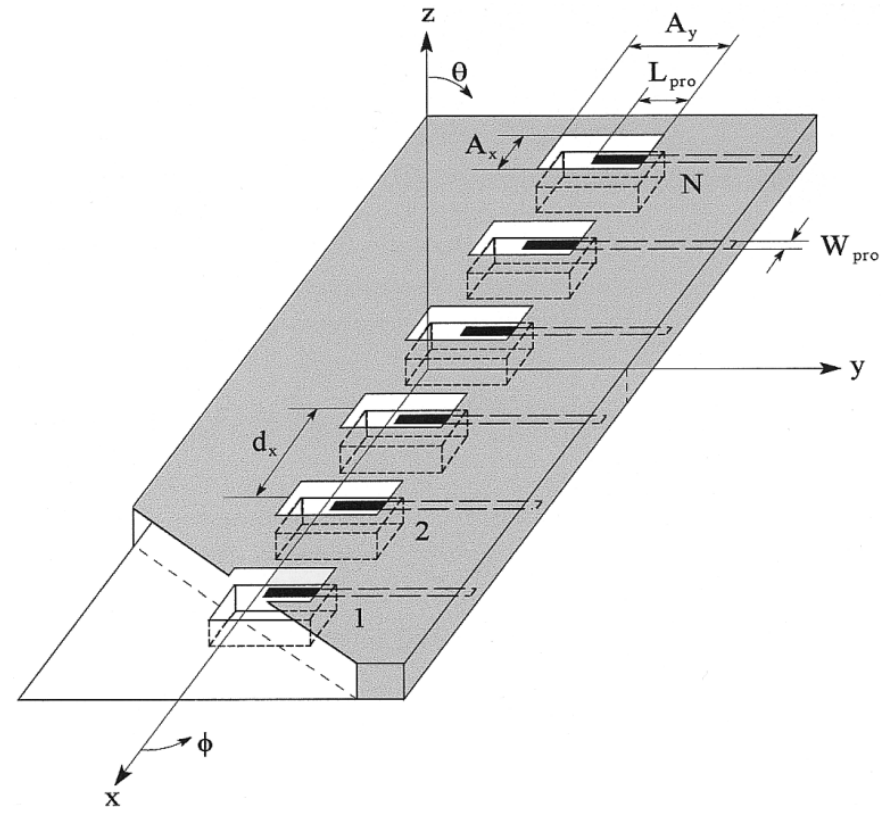

(a)

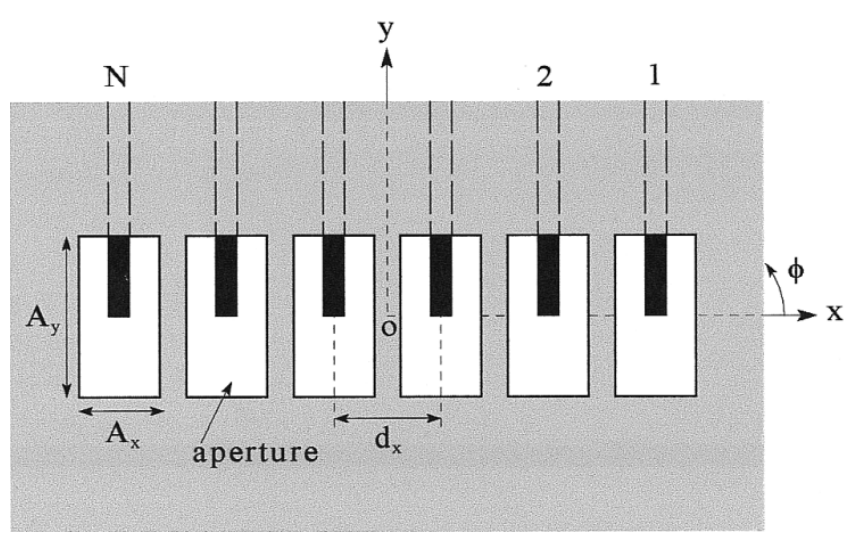

(b)

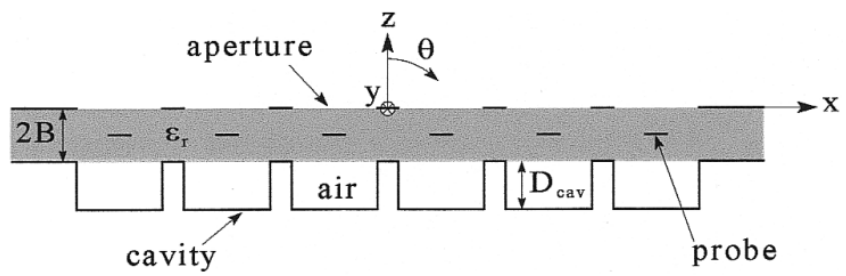

(c)

Fig. 9. Configuration of an array antenna composed of $N$ rectangular apertures. (a) Perspective view. (b) Top view. (c) Cross-sectional view.

Final investigation is directed toward the input impedance. The analysis shows that each array element has almost the same frequency response of the input impedance $Z_{\text {in }}$ as the single (isolated) aperture antenna discussed in Section III (see Fig. 7).

\section{CONCLUSION}

A rectangular aperture antenna based on the TTL structure is analyzed by using the FDTD method. The aperture cut in the top plate of the TTL, which has x-side length $A_{x}$ and y-side length $A_{y}$, is excited with a probe that is an extension of the inner conductor of the TTL. The bottom plate of the TTL has a cavity to obtain a tilted beam with high radiation efficiency. 


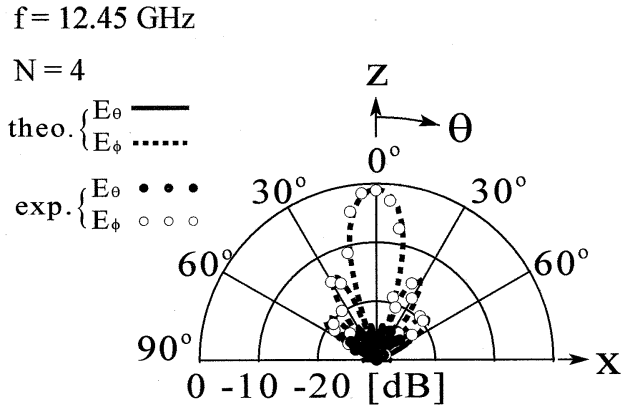

(a)

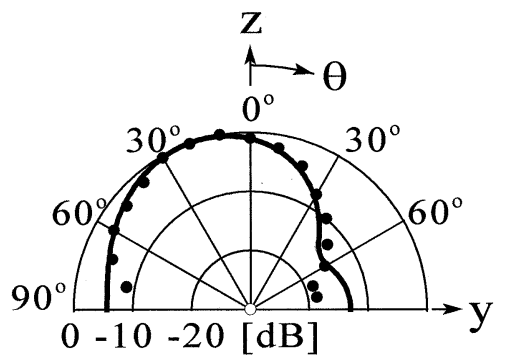

(b)

Fig. 10. Radiation patterns of a four-element array antenna. (a) In the $x-z$ plane. (b) In the $y-z$ plane.

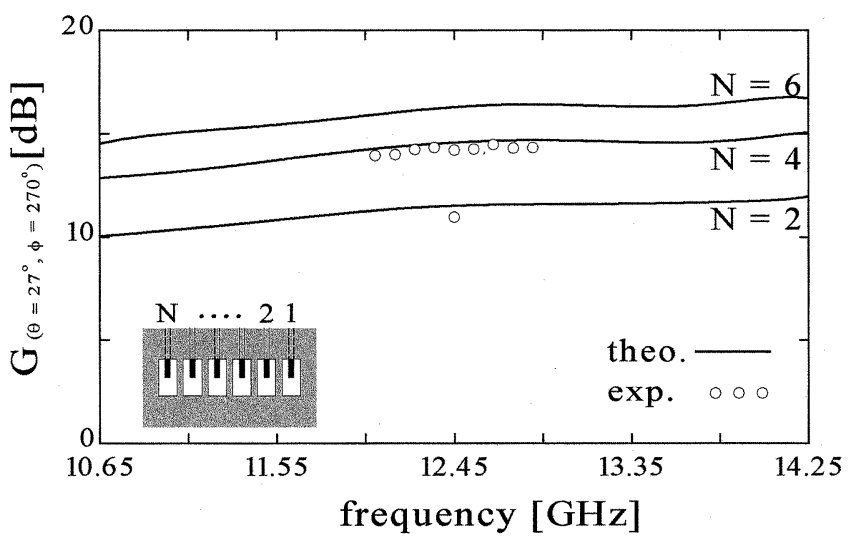

Fig. 11. Gain in the fixed direction $\left(\theta=27^{\circ}, \phi=270^{\circ}\right)$ as a function of frequency; the number of array elements is $N=2,4$, and 6 .

The Poynting vector distribution above the aperture shows that the antenna forms a tilted radiation beam. As the aspect ratio $A_{x} / A_{y}$ increases, the tilt angle increases. A tilt angle of approximately $27^{\circ}$ is obtained for $A_{x} / A_{y}=8 / 9=0.89$. The radiation efficiency $\eta_{\text {rad }}$ for a tilt angle of $\theta_{t}=27^{\circ}$ is calculated to be $87 \%$, which is higher than the radiation efficiency of an aperture antenna without a cavity (a FBS) by approximately $21 \%$. The gain in the beam direction $\left(\theta_{t}=27^{\circ}, \phi=270^{\circ}\right)$ for the cavity-backed aperture antenna with $A_{x} / A_{y}=8 / 9$ is approximately $8 \mathrm{~dB}$.

An aperture array antenna is also investigated, where aperture elements, each having an aspect ratio of $A_{x} / A_{y}=8 / 9$, are arrayed in the $\mathrm{x}$-direction with an array distance of $d_{x}=0.79$ wavelength. The array realizes a tilted fan beam with an increased gain. It is found that each array element has almost the same frequency response of the input impedance $Z_{\text {in }}$ as the single (isolated) aperture antenna.

\section{ACKNOWLEDGMENT}

The authors would like to thank V. Shkawrytko for his assistance in the preparation of this paper.

\section{REFERENCES}

[1] R. M. Sorbello, A. I. Zaghloul, J. E. Effland, and D. F. Difonzo, "A highefficiency flat plate array for direct broadcast satellite applications," in Proc. of Europ. Microwave Conf., Sweden, Sept. 1988, pp. 295-299.

[2] H. Nakano, T. Unno, K. Nakayama, and J. Yamauchi, "FDTD analysis and measurement of aperture antennas based on the triplate transmission-line structure," IEEE Trans. Antennas Propagat., vol. 47, pp. 986-992, June 1999.

[3] K. Nakayama, A. Takayasu, and H. Nakano, "A triplate-type slot antenna backed by a cavity (XII)," in Proc. of 1998 Commun. Society Conf. IEICE, Japan, Sept. 1998, B-1-85.

[4] H. Nakano, M. Iwatsuki, and J. Yamauchi, "A rectangular aperture antenna with a tilted beam," in Dig. IEEE APS Symp., Salt Lake City, UT, July 2000, pp. 1466-1469.

[5] K. S. Yee, "Numerical solution of initial boundary value problems involving Maxwell's equations in isotropic media," IEEE Trans. Antennas Propagat., vol. 14, pp. 302-307, May 1966.

[6] A. Taflove, Computational Electrodynamics: The Finite-Difference Time Domain Method. Norwood, MA: Artech House, 1995.

[7] Z. P. Liao, H. L. Wong, B. P. Yang, and Y. F. Yuan, "A transmitting boundary for transient wave analyzes," Science Sinica, ser. A, vol. 27, no. 10 , pp. 1063-1076, 1984.

[8] R. F. Harrington, Time-Harmonic Electromagnetic Fields. New York: McGraw-Hill, 1961, pp. 106-110.

[9] C. A. Balanis, Antenna Theory. New York: Harper and Row, 1982, ch. 11.

[10] Antenna Handbook, Van Nostrand Reinhold, Australia, 1988.

[11] H. Nakano, M. Sugama, and J. Yamauchi, "Cavity-backed circular aperture antenna constructed using a triplate transmission line," IEE Proc.Microw. Antennas Propag., vol. 146, no. 6, Dec. 1999.

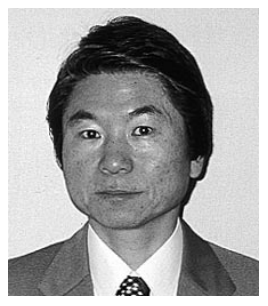

Hisamatsu Nakano (M'75-SM'87-F'92) received the B.E., M.E., and Dr. E. degrees in electrical engineering from Hosei University, Tokyo, in 1968, 1970, and 1974 , respectively.

Since 1973, he has been with the faculty of Hosei University, where he is now a Professor of electronic informatics. He has published more than 170 refereed journal papers and 140 international symposium papers on antenna and relevant problems.

Dr. Nakano received the Best Paper Award from the IEE 5th International Conference on Antennas and Propagation in 1987. In 1994, he received the IEEE AP-S Best Application Paper Award (H.A. Wheeler Award). He is an Associate Editor of IEEE Antennas and Propagation Magazine and a member of the AP-S administrative committee.

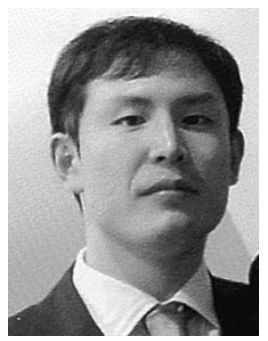

Masatoshi Iwatsuki received the B.E. and M.E. degrees in electrical engineering from Hosei University, Tokyo, Japan, in 1999 and 2001, respectively.

He joined Fujitsu Ltd., in 2001.

Mr. Iwatsuki is a Member of the Institute of Electronics, Information, and Communication Engineers (IEICE) of Japan. 


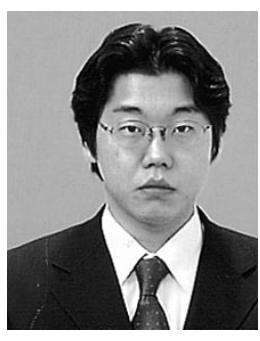

Masanori Sakurai was born in Chiba, Japan, on March 16, 1978. He received the B.E. degree in electronic informatics from Hosei University, Tokyo, Japan, in 2001.

$\mathrm{He}$ is currently pursuing the Master's degree at Hosei University. His current research interests are aperture antennas and spiral antennas for mobile and satellite communication systems.

Mr. Sakurai is a Member of the Institute of Electronics, Information, and Communication Engineers (IEICE) of Japan.

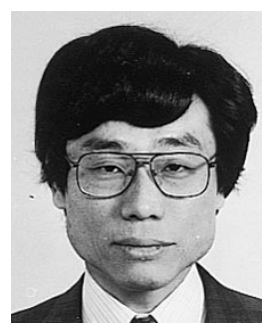

Junji Yamauchi (M'85) was born in Nagoya, Japan, on August 23, 1953. He received the B.E., M.E., and Dr. E. degrees from Hosei University, Tokyo, Japan, in 1976,1978 , and 1982, respectively.

From 1984 to 1988, he served as a Lecturer in the Electrical Engineering Department of Tokyo Metropolitan Technical College. Since 1988, he has been with the faculty of Hosei University, where he is now a Professor of electronic informatics. His research interests include optical waveguides and circularly polarized antennas. He is the author of Propagating Beam Analysis of Optical Waveguides (England: Research Studies, 2002)

Dr. Yamauchi is a Member of the Optical Society of America (OSA) and the Institute of Electronics, Information and Communication Engineers (IEICE) of Japan. 